\title{
Aortic arch surgery with hypothermic circulatory arrest and unilateral antegrade cerebral perfusion: Perioperative outcomes
}

\author{
Xiaomeng Wang, MD, ${ }^{\mathrm{a}}$ Feng Yang, MD, ${ }^{\mathrm{a}}$ Junming Zhu, MD, ${ }^{\mathrm{b}}$ Yongmin Liu, MD, ${ }^{\mathrm{b}}$ Lizhong Sun, MD, ${ }^{\mathrm{b}}$ and \\ Xiaotong Hou, MD, $\mathrm{PhD}^{\mathrm{a}}$
}

\begin{abstract}
Objective: The study objective was to determine the effects of surgical procedures, circulatory management strategies, and cerebral protection strategies on the short-term outcomes of aortic arch surgery based on the 7-year clinical experience of a single center.
\end{abstract}

Methods: We analyzed the data of 1708 patients who underwent aortic arch surgery with definite hypothermic circulatory arrest and unilateral antegrade cerebral perfusion at Beijing Anzhen Hospital between 2009 and 2015. Logistic regression and random Forest regression analyses were used to determine predictors and their effects on outcomes.

Results: Thirty-day mortality was $6.1 \%$. Permanent neurologic dysfunction incidence was $4.8 \%$. The proportion of patients requiring continuous renal replacement therapy was $7.9 \%$. In multivariable analyses, age, DeBakey type I dissection, New York Heart Association score, coma, coronary artery bypass grafting, extra-anatomic bypass, and cardiopulmonary bypass time were independent risk factors for mortality. Age, DeBakey type I dissection, and cardiopulmonary bypass time were independent risk factors for permanent neurologic dysfunction. In the random Forest regression, the risk for permanent neurologic dysfunction and mortality increased when unilateral antegrade cerebral perfusion time was more than 38 minutes and decreased with an increase in nasopharyngeal temperature when temperature was lower than approximately $24^{\circ} \mathrm{C}$. The risk for permanent neurologic dysfunction, continuous renal replacement therapy, and paraplegia increased when temperature was greater than approximately $24^{\circ} \mathrm{C}$.

Conclusions: The study showed that the largest reported cohort of patients undergoing aortic arch surgery with hypothermic circulatory arrest and unilateral antegrade cerebral perfusion had reasonable morbidity and mortality rates. As a cerebral protection strategy, unilateral antegrade cerebral perfusion may have a 38-minute safety threshold. Moderate hypothermia should be maintained below $24^{\circ} \mathrm{C}$ to reduce the risk for permanent neurologic dysfunction, paraplegia, and acute renal dysfunction requiring continuous renal replacement therapy. (J Thorac Cardiovasc Surg 2020;159:374-87)

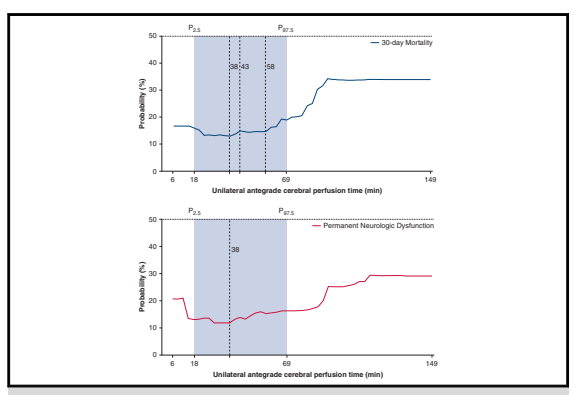

Probability for end points with prolonged unilateral antegrade cerebral perfusion times.

\section{Central Message}

This study explored the surgical experience and short-term outcomes of 1708 patients who underwent aortic arch surgery with unilateral antegrade cerebral perfusion at Beijing Anzhen Hospital.

\section{Perspective}

By reporting the short-term outcomes of the largest cohort of patients undergoing aortic arch surgery with hypothermic circulatory arrest and unilateral antegrade cerebral perfusion, this study seeks to provide insight into the relationship between circulatory management or cerebral protection strategies and clinical outcomes and to guide surgeons in choosing optimal intraoperative strategies.

See Commentaries on pages 388 and 390.
Surgical treatment of complex aortic diseases, such as acute dissection and aneurysms involving the transverse arch, remains one of the most technically challenging endeavors

From the ${ }^{\mathrm{a} C e n t e r}$ for Cardiac Intensive Care and ${ }^{\mathrm{b}}$ Beijing Aortic Disease Center, Beijing Institute of Heart, Lung and Blood Vessel Diseases, Beijing Anzhen Hospital, Capital Medical University, Beijing, China.

Funded by The National Key Research and Development Program of China (2016YFC1301001, to X.H.)

Received for publication April 15, 2018; revisions received Jan 24, 2019; accepted for publication Jan 31, 2019; available ahead of print March 15, 2019 of modern aortic surgery. Although surgical procedures have evolved significantly, they still carry a high risk of morbidity and mortality. ${ }^{1}$ Permanent neurologic

\footnotetext{
Address for reprints: Xiaotong Hou, MD, PhD, Center for Cardiac Intensive Care, and Lizhong Sun, MD, Beijing Aortic Disease Center, Beijing Institute of Heart, Lung and Blood Vessel Diseases, Beijing Anzhen Hospital, Capital Medical University, No 2 Anzhen Rd, Chaoyang District, Beijing, 100029, China (E-mail: houxiaotong_2013@163.com and lizhongsun@outlook.com). $0022-5223 / \$ 36.00$

Copyright (C) 2019 by The American Association for Thoracic Surgery https://doi.org/10.1016/j.jtcvs.2019.01.127
} 

Abbreviations and Acronyms
$\mathrm{CABG}=$ coronary artery bypass grafting
$\mathrm{CPB}=$ cardiopulmonary bypass
CRRT $=$ continuous renal replacement therapy
LBCA $=$ lower-body circulatory arrest
NYHA $=$ New York Heart Association
$\mathrm{OR}=$ odds ratio
RFR $=$ random Forest regression

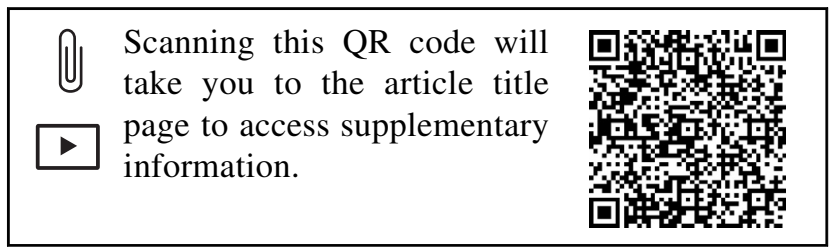

dysfunction is the most common complication and the leading cause of death associated with these procedures. ${ }^{1}$ Over the past 2 decades, surgeons have continued to optimize surgical procedures, circulatory management, and cerebral protection strategies. To date, the choice of surgical procedure, circulatory management strategy, and cerebral protection strategy in patients who require aortic arch surgery remain controversial in many centers with extensive surgical experience. ${ }^{2-4}$ There is a scarcity of studies that report the relationship between these factors and clinical outcomes in large cohorts of patients undergoing aortic arch surgery.

The present retrospective study sought to document the demographic data and clinical outcomes of patients who have undergone aortic arch surgery in our center, as well as the surgical procedures and circulatory management strategies used in these patients, and to determine risk factors for 30-day mortality, postoperative permanent neurologic dysfunction, and acute renal dysfunction requiring continuous renal replacement therapy (CRRT).

\section{MATERIALS AND METHODS}

This study was approved by the Institutional Review Board of Beijing Anzhen Hospital, Capital Medical University (No. 2017016X).

\section{Data Collection}

We conducted a retrospective analysis of data collected prospectively (from January 1, 2009, to January 1, 2016) at the medical center for aortic disease of Anzhen Hospital in Beijing. The preoperative and intraoperative data of a consecutive series of 1861 adult patients who underwent aortic arch surgery were collected. We excluded patients who (1) underwent hypothermic circulatory arrest alone $(\mathrm{n}=148)$ or hypothermic circulatory arrest with bilateral antegrade cerebral perfusion $(\mathrm{n}=2)$ or $(2)$ were pregnant $(\mathrm{n}=3)$. We analyzed the remaining data of 1708 patients who underwent aortic arch surgery with definite hypothermic circulatory arrest and unilateral antegrade cerebral perfusion during this period.

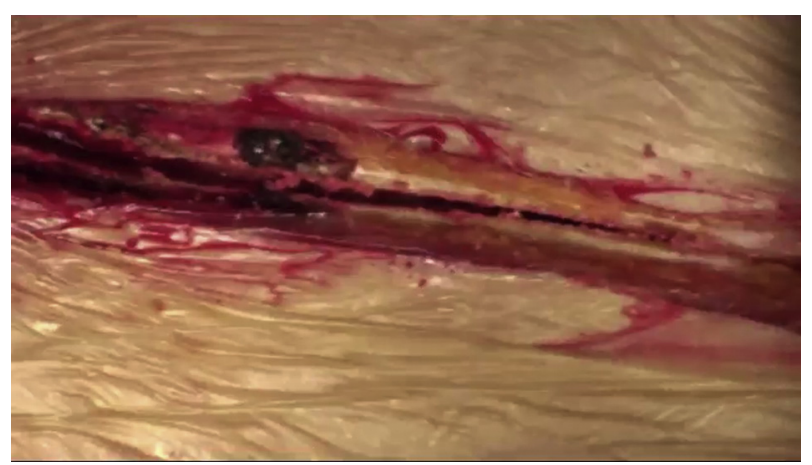

VIDEO 1. Total arch replacement and frozen elephant trunk. Video available at: https://www.jtcvs.org/article/S0022-5223(19)30362-9/fulltext.

\section{Surgical Techniques}

The procedures were selected on the basis of the surgical indications of the patients included in the study. Surgical indications and procedures are as follows: Total arch replacement was performed in patients with type A dissection if (1) the primary tear was in the transverse arch or the descending aorta; (2) an aneurysm was present in the aortic arch or the distal aorta (aneurysm size $\geq 40 \mathrm{~mm}$ ); (3) the brachiocephalic artery was involved or presented with aneurysm or occlusion; or (4) the patient had Marfan syndrome. If aortic dissection extended beyond the distal arch, a frozen elephant trunk was implanted into the distal aorta. Total arch replacement was not performed in patients with type A dissection with the primary tear in the ascending aorta without the features mentioned; these patients underwent only hemiarch replacement and ascending aortic replacement. ${ }^{5}$

The procedure of total arch replacement and frozen elephant trunk is shown in Video 1. In all cases, general anesthesia was induced with the patient in the supine position. Blood pressure in the left radial artery and left femoral artery was monitored. All procedures were performed using a median sternotomy and total cardiopulmonary bypass (CPB) with unilateral antegrade cerebral perfusion. The right axillary artery was the preferred inflow site for CPB and unilateral antegrade cerebral perfusion. After heparinization, CPB was initiated with a flow of 2.0 to $2.4 \mathrm{~L} / \mathrm{m}^{2} / \mathrm{min}$, and systemic cooling was used to induce hypothermia. Arterial blood $\mathrm{pH}$ was managed with an alpha-stat strategy. During the cooling phase, the ascending aorta (and the aortic root or valve in some patients) was replaced and other concomitant procedures were performed, if indicated. When the nasopharyngeal temperature reached $18^{\circ} \mathrm{C}$ to $28^{\circ} \mathrm{C}$, the 3 vessels of the arch were crossclamped (the arch vessels were also crossclamped in hemiarch replacement to prevent residual blood from interfering with the procedure) and lower-body circulatory arrest (LBCA) with unilateral antegrade cerebral perfusion was instituted. The cerebral perfusion flow rate was controlled at 5 to $10 \mathrm{~mL} / \mathrm{kg} / \mathrm{min}$, allowing for a perfusion pressure on the pump at 50 to $80 \mathrm{~mm} \mathrm{Hg}$. During the frozen elephant trunk procedure and total arch replacement using a 4 branched graft, distal reperfusion was initiated once the distal anastomosis was completed to minimize the duration of cerebral and spinal cord ischemia. The left carotid artery was reconstructed first (after which rewarming was initiated and the brain was perfused bilaterally), followed by the ascending aorta (to resume myocardial perfusion). The left subclavian artery was repaired, and, last, the innominate artery was repaired. After completion of the repair and adequate rewarming, the patient was weaned from $\mathrm{CPB}$, and the perfusion limb of the tetrafurcated graft was ligated and divided. The remainder of the procedure, including securing hemostasis and sternal closure, was performed in a routine manner. ${ }^{6}$

\section{Definitions}

The primary outcome measure was 30-day mortality. The secondary outcomes included in-hospital mortality and postoperative complications. Postoperative permanent neurologic dysfunction included stroke and 
TABLE 1. Preoperative and intraoperative conditions of all patients

\begin{tabular}{|c|c|}
\hline Name & $\begin{array}{c}\text { Total } \\
\mathbf{N}=\mathbf{1 7 0 8}\end{array}$ \\
\hline Sex (male) & $1282(75.1)$ \\
\hline Age (y) & $47(40-56)$ \\
\hline Body mass index $\left(\mathrm{kg} / \mathrm{m}^{2}\right)$ & $25.1(22.9-27.4)$ \\
\hline \multicolumn{2}{|l|}{ Pathological type } \\
\hline Aortic dissection & 1547 (90.6) \\
\hline Acute & $893(57.8)$ \\
\hline Time onset to operation (d) & $2(1-6)$ \\
\hline Time onset to diagnosis (d) & $2(1-3)$ \\
\hline Subacute & $273(17.6)$ \\
\hline Chronic & $381(24.6)$ \\
\hline \multicolumn{2}{|l|}{ DeBakey } \\
\hline I & 1339 (86.6) \\
\hline II & $208(13.4)$ \\
\hline Aortic aneurysm & $135(7.9)$ \\
\hline Pseudoaneurysm & $26(1.5)$ \\
\hline \multicolumn{2}{|l|}{ NYHA score } \\
\hline I & $45(2.6)$ \\
\hline II & $682(39.9)$ \\
\hline III & $912(53.4)$ \\
\hline IV & $69(4.0)$ \\
\hline Left ventricular ejection fraction & $63(58-68)^{*}$ \\
\hline Coronary artery disease & $136(8.0)$ \\
\hline Hypertension & $1242(72.7)$ \\
\hline Diabetes mellitus & $61(3.6)$ \\
\hline Chronic kidney disease & $39(2.3)$ \\
\hline Marfan syndrome & $52(3.0)$ \\
\hline Pericardial tamponade & $16(0.9)$ \\
\hline Acute heart failure & $12(0.7)$ \\
\hline Coma & $3(0.2)$ \\
\hline Lower-limb malperfusion & $154(9.0)$ \\
\hline Tracheotomy & $5(0.3)$ \\
\hline CRRT & $7(0.4)$ \\
\hline Previous stroke & $59(3.5)$ \\
\hline Redo sternotomy & $141(8.3)$ \\
\hline \multicolumn{2}{|l|}{ Cannulation site } \\
\hline Right axillary artery & 1349 (79.0) \\
\hline Innominate artery & $359(21.0)$ \\
\hline \multicolumn{2}{|l|}{ Main surgical procedure } \\
\hline Hemiarch replacement & $251(14.7)$ \\
\hline Total arch replacement & $38(2.2)$ \\
\hline $\begin{array}{l}\text { Frozen elephant trunk and total } \\
\text { arch replacement }\end{array}$ & $1419(83.1)$ \\
\hline \multicolumn{2}{|l|}{ Concomitant procedures } \\
\hline Ascending aorta replacement & 849 (49.7) \\
\hline Bentall & $637(37.3)$ \\
\hline CABG & $105(6.1)$ \\
\hline Valve surgery & $62(3.6)$ \\
\hline
\end{tabular}

TABLE 1. Continued

\begin{tabular}{lc}
\hline \multicolumn{1}{c}{ Name } & $\begin{array}{c}\text { Total } \\
\mathbf{N}=\mathbf{1 7 0 8}\end{array}$ \\
\hline Extra-anatomic bypass & $119(7.0)$ \\
Ascending aorta other arteries & 42 \\
Left common carotid other arteries & 37 \\
Left subclavian other arteries & 24 \\
Bilateral femoral arteries & 10 \\
Innominate other arteries & 3 \\
$\quad$ Bilateral axillary arteries & 3 \\
Nasopharyngeal temperature $\left({ }^{\circ} \mathrm{C}\right)$ & $23.2(22.0-24.2)$ \\
Rectal temperature $\left({ }^{\circ} \mathrm{C}\right)$ & $25.5(24.2-26.8) \dagger$ \\
Flow (mL/kg/min) & $5.9 \pm 1.8$ \\
Times & \\
Unilateral antegrade cerebral perfusion time (min) & $37(29-46)$ \\
Crossclamp time (min) & $97(78-123)$ \\
CPB time (min) & $181(155-212)$ \\
LBCA time (min) & $24(19-31)$ \\
Rewarming time (min) & $81(68-100)$ \\
\hline
\end{tabular}

Data presented as mean \pm standard deviation or median and interquartile range $(25 \%$, quartile 1 to $75 \%$, quartile 3) for continuous variables and $\mathrm{n}(\%)$ for categoric variables. NYHA, New York Heart Association; CRRT, continuous renal replacement therapy; $C A B G$, coronary artery bypass grafting; $C P B$, cardiopulmonary bypass; $L B C A$, lower-body circulatory arrest. *Available for 1657 patients. $\dagger$ Available for 1696 patients.

paraplegia during hospitalization. Stroke was defined as the presence of permanent neurologic deficits that consisted of abnormal movement of limbs, coma, epilepsy, and sensory loss affecting one side of the body, with confirmation of the diagnosis by a neurologist or correlation of the clinical diagnosis with morphologic changes revealed by neuroimaging examination. If patients had relevant neurologic dysfunction before surgery, stroke was defined as any newly developed sensorimotor deficit persisting at the time of discharge confirmed by neurologic consultations and neuroimaging examinations. ${ }^{3,7}$ Paraplegia was defined as numbness, weakness, or bilateral sensory dysfunction of the lower extremities that did not heal until hospital discharge. Patients who received preoperative CRRT were excluded from the assessment of postoperative CRRT data.

Unilateral antegrade cerebral perfusion time was defined as the period from the initiation of unilateral antegrade cerebral perfusion to bilateral antegrade cerebral perfusion. LBCA time was defined as the period from the initiation of LBCA to the restoration of distal aortic perfusion.

\section{Statistical Analysis}

Continuous variables were presented as median (interquartile range). Categoric variables were described as frequencies (\%). Missing data are shown in Table 1. Random Forest imputation was used to maximize use of available data (details in Appendix E1).

Risk factors for outcomes were identified by univariate and multivariable logistic regression analyses with SPSS for Windows version 22.0 (SPSS, Chicago, Ill). All clinically relevant variables with $P$ less than .100 on univariate analysis except for main surgical procedure were incorporated into the multivariable logistic models directly because of the obvious collinearity between main surgical procedure and DeBakey type. The tests for collinearity of variables and the accuracy of models are shown in Tables E1 to E6.

We used random Forest regression (RFR) with R software 3.5.1 (www.rproject.org) as the analytic strategy in part to avoid restrictive parametric modeling assumptions, given no prior knowledge of what relationships might exist, and in part because some studies previously demonstrated 
with this method that there is a complex interplay between characteristics of diseases and outcomes (details in Appendix E1). Briefly, a random Forest is a collection of decision-tree analyses, wherein a variable is chosen to optimally split the population to improve prediction. This process is applied recursively to create a tree (recursive partitioning, classification, and regression trees). Individual trees "grown" by this method are inherently unstable, which can be mitigated by creating a collection of trees from bootstrap samples of the original data set. Subsequently, an ensemble average can be formulated across this Forest of individual trees. Validity of the Forest is evaluated by assessing outcomes of patients who were not selected in the bootstrap process $(37 \%)$, resulting in internal multifold cross validation. This transforms variables associated with an outcome of interest into predictors of that outcome.

A random Forest was constructed with 1500 trees by the "randomForest" package. At each node, all variables (the day of operation, sex, age, body mass index, pathological DeBakey type, New York Heart Association [NYHA] score, left ventricular ejection fraction, coronary artery disease, hypertension, diabetes mellitus, chronic kidney disease, Marfan syndrome, pericardial tamponade, acute heart failure, coma, lower-limb malperfusion, tracheotomy, previous CRRT, previous stroke, redo sternotomy, cannulation site of unilateral antegrade cerebral perfusion, main surgery procedure, ascending aorta replacement, Bentall, coronary artery bypass grafting [CABG], valve surgery, extra-anatomic bypass, nasopharyngeal temperature, rectal temperature, cerebral perfusion flow, unilateral antegrade cerebral perfusion time, crossclamp time, CPB, LBCA time, and rewarming time) were used, but 6 candidate variables were randomly selected from among these for splitting each node.

The variable importance was measured by the Gini index (details in Appendix E1). We used partial dependence to determine how variables were related to outcomes. Partial dependency adjusted for all variables in the model. Conditional partial dependence plots were used to detect interactions between the variables (LBCA time, unilateral antegrade cerebral perfusion time, and nasopharyngeal temperature) and the outcomes. ${ }^{8}$

\section{RESULTS}

\section{Demographic Characteristics}

A total of 1708 patients who underwent aortic arch surgery with definite hypothermic circulatory arrest and unilateral antegrade cerebral perfusion over the 7-year observation period were selected for this study. The demographic characteristics are listed in Table 1. The mean age of all patients was 47 years. Three-quarters of the patients were men. The aortic diseases affecting the patients were aortic dissection $(90.6 \%)$, aortic aneurysm $(7.9 \%)$, and pseudoaneurysm (1.5\%). In patients with aortic dissection, acute aortic dissection accounted for $57.8 \%$, subacute aortic dissection accounted for $17.6 \%$, and chronic aortic dissection accounted for $24.6 \%$ of the cases. DeBakey type I and II dissections accounted for $86.6 \%$ and $13.4 \%$ of dissection cases, respectively. Marfan syndrome was diagnosed in $3.0 \%$ of patients. A history of hypertension was reported in $72.7 \%$ of patients.

\section{Thirty-Day Mortality and Morbidity}

Thirty-day mortality was $6.1 \%(n=104)$. The incidence of permanent neurologic dysfunction was $4.8 \%(n=82)$. The incidences of stroke and paraplegia were $2.8 \%$ $(\mathrm{n}=48)$ and $2.0 \%(\mathrm{n}=34)$, respectively. The incidence of acute renal dysfunction requiring CRRT was $7.9 \%$
TABLE 2. Early outcomes of all patients

\begin{tabular}{lc}
\hline \multicolumn{1}{c}{ Name } & $\mathbf{N}=\mathbf{1 7 0 8}$ \\
\hline 30-d death & $104(6.1)$ \\
In-hospital death & $112(6.6)$ \\
Intraoperative death & 2 \\
Permanent neurologic dysfunction & 43 \\
Circulatory failure & 34 \\
Ventricular arrhythmia & 8 \\
Infection & 7 \\
Hepatic failure & 4 \\
Renal failure & 4 \\
Distal aneurysm rupture & 4 \\
Respiratory failure & 4 \\
Gastrointestinal hemorrhage & 1 \\
Lower osteofascial compartment syndrome & 1 \\
Permanent neurologic dysfunction & $82(4.8)$ \\
Stroke/coma & $48(2.8)$ \\
Paraplegia & $34(2.0)$ \\
CRRT & $135(7.9)$ \\
\hline Infection & $45(2.7)$ \\
Atrial fibrillation & $211(12.4)$ \\
\hline Ventricular fibrillation & $22(1.3)$ \\
Cardiac arrest & $13(0.8)$ \\
\hline Intra-aortic balloon pump & $3(0.2)$ \\
Extracorporeal membrane oxygenation & $10(0.6)$ \\
\hline Left heart bypass & $3(0.2)$ \\
Tracheotomy & $64(3.7)$ \\
Reexploration for bleeding & $102(6.0)$ \\
Mechanical ventilation time (h) & $18(14-43)$ \\
\hline Intensive care unit stay time (d) & $2(1-3)$ \\
\hline
\end{tabular}

Data presented as median and interquartile range ( $25 \%$, quartile 1 to $75 \%$, quartile 3$)$ for continuous variables and $\mathrm{n}(\%)$ for categoric variables. CRRT, Continuous renal replacement therapy.

$(n=135)$. In-hospital death and other postoperative complications are listed in Table 2.

\section{Risk Factors for 30-Day Mortality}

In multivariable logistic analysis, age (odds ratio [OR], 1.03; $P=.010)$, DeBakey type I dissection (OR, 3.26; $P=.009)$, NYHA score (OR, 2.28; $P<.001)$, coma (OR, 25.99; $P=.012)$, CABG (OR, 2.99; $P=.002)$, extraanatomic bypass (OR, 4.48; $P<.001)$, and CPB time (OR, 1.01; $P<.001)$ were independent risk factors for 30 day mortality (Table 3 ).

\section{Risk Factors for Permanent Neurologic Dysfunction}

In multivariable logistic analysis, age $(\mathrm{OR}, 1.04 ; P=.001)$, DeBakey type I dissection (OR, 4.74; $P=.005)$, and CPB time (OR, $1.01 ; P=.014)$ were independent risk factors for permanent neurologic dysfunction (Table 4). 
TABLE 3. Univariate and multivariable logistic regression analyses of 30-day mortality for all patients

\begin{tabular}{|c|c|c|c|c|}
\hline \multirow[b]{2}{*}{ Name } & \multicolumn{2}{|c|}{ Univariate } & \multicolumn{2}{|c|}{ Multivariable } \\
\hline & OR $(95 \%$ CI $)$ & $P$ & OR $(95 \%$ CI $)$ & $P$ \\
\hline Day of operation (d) & $1.00(1.00-1.00)$ & .669 & & \\
\hline Sex (male) & $1.26(0.77-2.04)$ & .358 & & \\
\hline Age (y) & $1.02(1.00-1.04)$ & .020 & $1.03(1.01-1.05)$ & .010 \\
\hline BMI $\left(\mathrm{kg} / \mathrm{m}^{2}\right)$ & $1.01(0.95-1.06)$ & .853 & & \\
\hline Acute aortic dissection & $1.71(1.13-2.58)$ & .011 & $1.12(0.69-1.83)$ & .646 \\
\hline DeBakey (I) & $3.88(1.79-8.44)$ & .001 & $3.26(1.35-7.87)$ & .009 \\
\hline NYHA & $2.37(1.68-3.35)$ & $<.001$ & $2.28(1.57-3.32)$ & $<.001$ \\
\hline LVEF & $0.98(0.96-1.01)$ & .197 & & \\
\hline Coronary artery disease & $1.72(0.94-3.16)$ & .081 & $0.75(0.34-1.65)$ & .475 \\
\hline Hypertension & $1.20(0.75-1.90)$ & .444 & & \\
\hline Diabetes mellitus & $1.08(0.39-3.05)$ & .876 & & \\
\hline Chronic kidney disease & $2.92(1.19-7.12)$ & .019 & $1.54(0.48-4.88)$ & .466 \\
\hline Pericardial tamponade & $3.64(1.02-12.96)$ & .047 & $1.53(0.27-8.70)$ & .634 \\
\hline Acute heart failure & $1.41(0.18-11.00)$ & .745 & & \\
\hline Coma & $7.77(0.70-86.47)$ & .095 & $25.99(2.07-326.37)$ & .012 \\
\hline Lower-limb malperfusion & $1.10(0.55-2.12)$ & .826 & & \\
\hline Tracheotomy & $3.88(0.43-35.06)$ & .227 & & \\
\hline CRRT & $6.27(1.20-32.72)$ & .029 & $4.34(0.54-35.05)$ & .169 \\
\hline Previous stroke & $1.13(0.40-3.17)$ & .821 & & \\
\hline Redo sternotomy & $1.49(0.80-2.79)$ & .212 & & \\
\hline \multicolumn{5}{|l|}{ Cannulation site } \\
\hline Right axillary artery & $0.79(0.50-1.25)$ & .305 & & \\
\hline Innominate artery & $1.27(0.80-2.02)$ & .305 & & \\
\hline Main surgical procedure* & & .044 & - & - \\
\hline Hemiarch replacement & $0.40(0.18-0.86)$ & .020 & & \\
\hline TAR & $0.37(0.05-2.74)$ & .332 & & \\
\hline FET and TAR & - & - & & \\
\hline \multicolumn{5}{|l|}{ Concomitant procedures } \\
\hline Ascending aorta replacement & $1.19(0.80-1.78)$ & .384 & & \\
\hline Bentall & $1.10(0.73-1.65)$ & .643 & & \\
\hline $\mathrm{CABG}$ & $4.26(2.49-7.26)$ & $<.001$ & $2.99(1.48-6.06)$ & .002 \\
\hline Valve surgery & $1.69(0.71-4.03)$ & .234 & & \\
\hline Extra-anatomic bypass & $2.02(1.09-3.73)$ & .025 & $4.48(2.13-9.42)$ & $<.001$ \\
\hline Nasopharyngeal temperature $\left({ }^{\circ} \mathrm{C}\right)$ & $0.88(0.80-0.98)$ & .018 & $0.95(0.82-1.10)$ & .515 \\
\hline Rectal temperature $\left({ }^{\circ} \mathrm{C}\right)$ & $0.87(0.80-0.95)$ & .003 & $0.96(0.84-1.09)$ & .511 \\
\hline Flow (mL/kg/min) & $0.97(0.87-1.09)$ & .623 & & \\
\hline \multicolumn{5}{|l|}{ Times } \\
\hline UACP time (min) & $1.03(1.02-1.04)$ & $<.001$ & $1.01(0.99-1.03)$ & .307 \\
\hline Crossclamp time (min) & $1.01(1.01-1.02)$ & $<.001$ & $1.00(0.99-1.01)$ & .729 \\
\hline CPB time (min) & $1.01(1.01-1.02)$ & $<.001$ & $1.01(1.01-1.01)$ & $<.001$ \\
\hline LBCA time (min) & $1.01(0.99-1.03)$ & .105 & & \\
\hline Rewarming time (min) & $1.02(1.01-1.02)$ & $<.001$ & $1.00(0.99-1.01)$ & .947 \\
\hline
\end{tabular}

$O R$, Odds ratio; $C I$, confidence interval; $B M I$, body mass index; $N Y H A$, New York Heart Association; $L V E F$, left ventricular ejection fraction; $C R R T$, continuous renal replacement therapy; $T A R$, total arch replacement; $F E T$, frozen elephant trunk; $C A B G$, coronary artery bypass grafting; $U A C P$, unilateral antegrade cerebral perfusion; $C P B$, cardiopulmonary bypass; $L B C A$, lower-body circulatory arrest. *Main surgical procedure was removed from the final multivariable model because of the collinearity between main surgical procedure and DeBakey type. 
TABLE 4. Univariate and multivariable logistic regression analyses of permanent neurologic dysfunction for all patients

\begin{tabular}{|c|c|c|c|c|}
\hline \multirow[b]{2}{*}{ Name } & \multicolumn{2}{|c|}{ Univariate } & \multicolumn{2}{|c|}{ Multivariable } \\
\hline & OR $(95 \%$ CI $)$ & $P$ & OR $(95 \%$ CI $)$ & $P$ \\
\hline Day of operation (d) & $1.00(1.00-1.00)$ & .038 & $1.00(1.00-1.00)$ & .080 \\
\hline Sex (male) & $1.11(0.66-1.87)$ & .704 & & \\
\hline Age $(y)$ & $1.04(1.02-1.06)$ & .001 & $1.04(1.02-1.06)$ & .001 \\
\hline $\operatorname{BMI}\left(\mathrm{kg} / \mathrm{m}^{2}\right)$ & $1.01(0.95-1.07)$ & .853 & & \\
\hline Acute aortic dissection & $1.81(1.13-2.89)$ & .013 & $1.07(0.63-1.80)$ & .800 \\
\hline DeBakey (I) & $5.43(1.97-14.93)$ & .001 & $4.74(1.62-13.90)$ & .005 \\
\hline NYHA & $1.52(1.05-2.19)$ & .027 & $1.25(0.85-1.85)$ & .260 \\
\hline LVEF & $0.99(0.96-1.02)$ & .470 & & \\
\hline Coronary artery disease & $1.65(0.83-3.28)$ & .151 & & \\
\hline Hypertension & $1.71(0.97-3.03)$ & .064 & $1.23(0.67-2.24)$ & .509 \\
\hline Diabetes mellitus & $1.03(0.32-3.35)$ & .965 & & \\
\hline Chronic kidney disease & $1.07(0.25-4.53)$ & .923 & & \\
\hline Lower limb malperfusion & $1.26(0.62-2.57)$ & .526 & & \\
\hline Tracheotomy & $5.01(0.55-45.30)$ & .152 & & \\
\hline Previous stroke & $2.34(0.98-5.62)$ & .056 & $2.04(0.80-5.18)$ & .133 \\
\hline Redo sternotomy & $0.71(0.28-1.79)$ & .469 & & \\
\hline \multicolumn{5}{|l|}{ Cannulation site } \\
\hline Right axillary artery & $0.57(0.30-1.08)$ & .084 & $1.41(0.72-2.75)$ & .316 \\
\hline Innominate artery & $1.76(0.92-3.35)$ & .087 & & \\
\hline Main surgical procedure* & & .023 & - & - \\
\hline Hemiarch replacement & $0.14(0.03-0.57)$ & .006 & & \\
\hline TAR & $0.96(0.23-4.04)$ & .950 & & \\
\hline FET and TAR & - & - & & \\
\hline \multicolumn{5}{|l|}{ Concomitant procedures } \\
\hline Ascending aorta replacement & $1.45(0.93-2.28)$ & .103 & & \\
\hline Bentall & $0.82(0.51-1.31)$ & .403 & & \\
\hline CABG & $2.83(1.48-5.40)$ & .002 & $1.95(0.93-4.07)$ & .077 \\
\hline Valve surgery & $0.65(0.16-2.72)$ & .557 & & \\
\hline Extra-anatomic bypass & $0.86(0.34-2.17)$ & .751 & & \\
\hline Nasopharyngeal temperature $\left({ }^{\circ} \mathrm{C}\right)$ & $0.91(0.81-1.02)$ & .096 & $0.89(0.76-1.05)$ & .181 \\
\hline Rectal temperature $\left({ }^{\circ} \mathrm{C}\right)$ & $0.90(0.82-1.00)$ & .044 & $0.97(0.85-1.12)$ & .686 \\
\hline Flow (mL/kg/min) & $0.97(0.85-1.10)$ & .600 & & \\
\hline \multicolumn{5}{|l|}{ Times } \\
\hline UACP time (min) & $1.01(1.00-1.03)$ & .048 & $1.00(0.98-1.02)$ & .853 \\
\hline Crossclamp time (min) & $1.01(1.00-1.01)$ & $<.001$ & $1.00(0.99-1.01)$ & .305 \\
\hline CPB time (min) & $1.01(1.01-1.01)$ & $<.001$ & $1.01(1.00-1.01)$ & .014 \\
\hline LBCA time (min) & $0.99(0.97-1.01)$ & .450 & & \\
\hline Rewarming time (min) & $1.01(1.00-1.02)$ & .002 & $1.00(0.99-1.01)$ & .594 \\
\hline
\end{tabular}

$O R$, Odds ratio; $C I$, confidence interval; $B M I$, body mass index; $N Y H A$, New York Heart Association; $L V E F$, left ventricular ejection fraction; TAR, total arch replacement; $F E T$, frozen elephant trunk; $C A B G$, coronary artery bypass grafting; $U A C P$, unilateral antegrade cerebral perfusion; $C P B$, cardiopulmonary bypass; $L B C A$, lower-body circulatory arrest. *Main surgical procedures were removed from the final multivariable model because of the collinearity between main surgical procedure and DeBakey type.

\section{Risk Factors for Paraplegia}

In multivariable logistic analysis, age (OR, 1.04; $P=.030)$ and DeBakey type I dissection (OR, 4.72; $P=.039)$ were independent risk factors for paraplegia (Table 5).

\section{Risk Factors for Acute Renal Dysfunction Requiring Continuous Renal Replacement Therapy}

In multivariable logistic analysis, the day of operation (OR, 1.001; $P=.007)$, age (OR, $1.03 ; P<.001)$, previous chronic kidney disease $(\mathrm{OR}, 7.82 ; P<.001)$, valve surgery 
TABLE 5. Univariate and multivariable logistic regression analyses of paraplegia for all patients

\begin{tabular}{|c|c|c|c|c|}
\hline \multirow[b]{2}{*}{ Name } & \multicolumn{2}{|c|}{ Univariate } & \multicolumn{2}{|c|}{ Multivariable } \\
\hline & OR $(95 \%$ CI $)$ & $P$ & OR $(95 \%$ CI $)$ & $P$ \\
\hline Day of operation (d) & $1.00(1.00-1.00)$ & .346 & & \\
\hline Sex (male) & $1.08(0.49-2.41)$ & .848 & & \\
\hline Age $(y)$ & $1.04(1.01-1.07)$ & .025 & $1.04(1.00-1.07)$ & .030 \\
\hline BMI $\left(\mathrm{kg} / \mathrm{m}^{2}\right)$ & $0.96(0.87-1.05)$ & .371 & & \\
\hline Acute aortic dissection & $1.31(0.66-2.61)$ & .442 & & \\
\hline DeBakey (I) & $4.32(1.03-18.12)$ & .045 & $4.72(1.08-20.58)$ & .039 \\
\hline NYHA & $1.01(0.58-1.75)$ & 975 & & \\
\hline LVEF & $0.97(0.93-1.02)$ & .206 & & \\
\hline Coronary artery disease & $3.11(1.33-7.27)$ & .009 & $1.72(0.63-4.68)$ & .292 \\
\hline Hypertension & $2.20(0.85-5.73)$ & .105 & & \\
\hline Diabetes mellitus & $1.71(0.40-7.31)$ & .469 & & \\
\hline Lower-limb malperfusion & $1.77(0.67-4.63)$ & .248 & & \\
\hline Previous stroke & $0.84(0.11-6.28)$ & .869 & & \\
\hline \multicolumn{5}{|l|}{ Cannulation site } \\
\hline Right axillary artery & $2.02(0.71-5.77)$ & .190 & & \\
\hline Innominate artery & $0.50(0.17-1.42)$ & .190 & & \\
\hline Main surgical procedure & & .222 & & \\
\hline Hemiarch replacement & $0.17(0.02-1.28)$ & .085 & & \\
\hline TAR & $1.17(0.16-8.80)$ & .878 & & \\
\hline FET and TAR & - & - & & \\
\hline \multicolumn{5}{|l|}{ Concomitant procedures } \\
\hline Ascending aorta replacement & $1.65(0.82-3.32)$ & .160 & & \\
\hline Bentall & $0.80(0.39-1.65)$ & .548 & & \\
\hline CABG & $4.17(1.77-9.81)$ & .001 & $2.64(0.95-7.30)$ & .062 \\
\hline Valve surgery & $1.68(0.39-7.18)$ & .483 & & \\
\hline Extra-anatomic bypass & $1.30(0.39-4.32)$ & .668 & & \\
\hline Nasopharyngeal temperature $\left({ }^{\circ} \mathrm{C}\right)$ & $0.98(0.81-1.17)$ & .794 & & \\
\hline Rectal temperature $\left({ }^{\circ} \mathrm{C}\right)$ & $0.98(0.84-1.14)$ & .755 & & \\
\hline Flow (mL/kg/min) & $0.96(0.79-1.18)$ & .700 & & \\
\hline \multicolumn{5}{|l|}{ Times } \\
\hline UACP time (min) & $1.00(0.98-1.03)$ & .986 & & \\
\hline Crossclamp time (min) & $1.01(1.00-1.01)$ & .063 & $1.00(0.99-1.01)$ & .861 \\
\hline CPB time (min) & $1.01(1.00-1.01)$ & .019 & $1.00(0.99-1.01)$ & .976 \\
\hline LBCA time (min) & $0.98(0.94-1.01)$ & .178 & & \\
\hline Rewarming time (min) & $1.01(1.00-1.02)$ & .019 & $1.00(0.99-1.02)$ & .501 \\
\hline
\end{tabular}

$\overline{O R}$, Odds ratio; $C I$, confidence interval; $B M I$, body mass index; $N Y H A$, New York Heart Association; $L V E F$, left ventricular ejection fraction; TAR, total arch replacement; $F E T$, frozen elephant trunk; $C A B G$, coronary artery bypass grafting; $U A C P$, unilateral antegrade cerebral perfusion; $C P B$, cardiopulmonary bypass; $L B C A$, lower-body circulatory arrest.

$(\mathrm{OR}, 2.78 ; P=.015)$, and $\mathrm{CPB}$ time $(\mathrm{OR}, 1.01 ; P=.001)$ were independent risk factors for CRRT (Table 6).

\section{Unilateral Antegrade Cerebral Perfusion Time}

In RFR, the variable importance is shown in Figure E1. We further analyzed associations of the unilateral antegrade cerebral perfusion time with the outcomes. When unilateral antegrade cerebral perfusion time was more than 38 minutes, an upward trend was observed in 30-day mortality. At the beginning, this trend was steep from 38 to 43 minutes and stayed at this level until 58 minutes. Then this trend became sharp again. Likewise, an upward trend was observed for permanent neurologic dysfunction when unilateral antegrade cerebral perfusion time was more than 38 minutes (Figure 1).

\section{Lower-Body Circulatory Arrest Time}

In the RFR, a sharp upward trend for paraplegia was observed when LBCA time was more than 40 minutes 
TABLE 6. Univariate and multivariable logistic regression analyses of continuous renal replacement therapy for all patients who underwent aortic arch surgery with hypothermic circulatory arrest and unilateral antegrade cerebral perfusion without previous continuous renal replacement therapy

\begin{tabular}{|c|c|c|c|c|}
\hline \multirow[b]{2}{*}{ Name } & \multicolumn{2}{|c|}{ Univariate } & \multicolumn{2}{|c|}{ Multivariable } \\
\hline & OR $(95 \%$ CI) & $P$ & OR $(95 \%$ CI) & $P$ \\
\hline Day of operation (d) & $1.00(1.00-1.01)$ & $<.001$ & $1.00(1.000-1.001)$ & .007 \\
\hline Sex (male) & $0.95(0.63-1.42)$ & .796 & & \\
\hline Age $(y)$ & $1.03(1.02-1.05)$ & $<.001$ & $1.03(1.02-1.05)$ & $<.001$ \\
\hline BMI $\left(\mathrm{kg} / \mathrm{m}^{2}\right)$ & $1.01(0.97-1.06)$ & .566 & & \\
\hline Acute aortic dissection & $2.14(1.47-3.12)$ & $<.001$ & $1.30(0.82-2.07)$ & .269 \\
\hline DeBakey (I) & $2.89(1.58-5.29)$ & .001 & $1.77(0.88-3.57)$ & .112 \\
\hline NYHA & $1.51(1.13-2.03)$ & .006 & $1.12(0.81-1.54)$ & .513 \\
\hline LVEF & $1.02(0.99-1.04)$ & .234 & & \\
\hline Coronary artery disease & $1.56(0.88-2.76)$ & .125 & & \\
\hline Hypertension & $1.83(1.15-2.91)$ & .010 & $1.35(0.80-2.28)$ & .270 \\
\hline Diabetes mellitus & $1.32(0.56-3.13)$ & .527 & & \\
\hline Chronic kidney disease & $10.54(5.22-21.29)$ & $<.001$ & $7.82(3.57-17.11)$ & $<.001$ \\
\hline Marfan syndrome & $0.73(0.22-2.36)$ & .596 & & \\
\hline Pericardial tamponade & $4.91(1.52-15.87)$ & .008 & $1.32(0.30-5.92)$ & .714 \\
\hline Coma & $6.02(0.54-66.87)$ & .144 & & \\
\hline Lower-limb malperfusion & $2.06(1.25-3.39)$ & .005 & $1.68(0.97-2.91)$ & .067 \\
\hline Tracheotomy & $3.01(0.33-27.11)$ & .326 & & \\
\hline Previous stroke & $1.11(0.44-2.83)$ & .823 & & \\
\hline Redo sternotomy & $1.01(0.53-1.93)$ & .966 & & \\
\hline \multicolumn{5}{|l|}{ Cannulation site } \\
\hline Right axillary artery & $1.02(0.66-1.58)$ & .934 & & \\
\hline Innominate artery & $0.98(0.63-1.53)$ & .934 & & \\
\hline Main surgical procedure* & & .028 & - & - \\
\hline Hemiarch replacement & $0.40(0.20-0.80)$ & .009 & & \\
\hline TAR & $0.60(0.14-2.51)$ & .482 & & \\
\hline FET and TAR & - & - & & \\
\hline \multicolumn{5}{|l|}{ Concomitant procedures } \\
\hline Ascending aorta replacement & $1.39(0.97-2.00)$ & .074 & $1.20(0.79-3.02)$ & .205 \\
\hline Bentall & $1.08(0.75-1.56)$ & 669 & & \\
\hline CABG & $2.54(1.46-4.42)$ & .001 & $1.54(0.79-3.02)$ & .205 \\
\hline Valve surgery & $2.46(1.22-4.96)$ & .012 & $2.78(1.22-6.30)$ & .015 \\
\hline Extra-anatomic bypass & $1.24(0.65-2.37)$ & .516 & & \\
\hline Nasopharyngeal temperature $\left({ }^{\circ} \mathrm{C}\right)$ & $0.95(0.87-1.04)$ & .263 & & \\
\hline Rectal temperature $\left({ }^{\circ} \mathrm{C}\right)$ & $0.92(0.85-0.99)$ & .032 & $0.93(0.83-1.03)$ & .137 \\
\hline Flow $(\mathrm{mL} / \mathrm{kg} / \mathrm{min})$ & $0.94(0.85-1.05)$ & .292 & & \\
\hline \multicolumn{5}{|l|}{ Times } \\
\hline UACP time (min) & $1.02(1.01-1.03)$ & $<.001$ & $1.00(0.97-1.02)$ & .906 \\
\hline Crossclamp time (min) & $1.02(1.01-1.02)$ & $<.001$ & $1.00(0.99-1.01)$ & .947 \\
\hline $\mathrm{CPB}$ time $(\mathrm{min})$ & $1.01(1.01-1.02)$ & $<.001$ & $1.01(1.01-1.02)$ & $<.001$ \\
\hline LBCA time (min) & $1.02(1.01-1.03)$ & .008 & $1.00(0.98-1.03)$ & .925 \\
\hline Rewarming time (min) & $1.01(1.01-1.02)$ & $<.001$ & $1.00(0.99-1.00)$ & .147 \\
\hline
\end{tabular}

$O R$, Odds ratio; $C I$, confidence interval; $B M I$, body mass index; $N Y H A$, New York Heart Association; $L V E F$, left ventricular ejection fraction; TAR, total arch replacement; $F E T$, frozen elephant trunk; $C A B G$, coronary artery bypass grafting; $U A C P$, unilateral antegrade cerebral perfusion; $C P B$, cardiopulmonary bypass; $L B C A$, lower-body circulatory arrest. *Main surgical procedures were removed from the final multivariable model because of the collinearity between main surgical procedure and DeBakey type. 

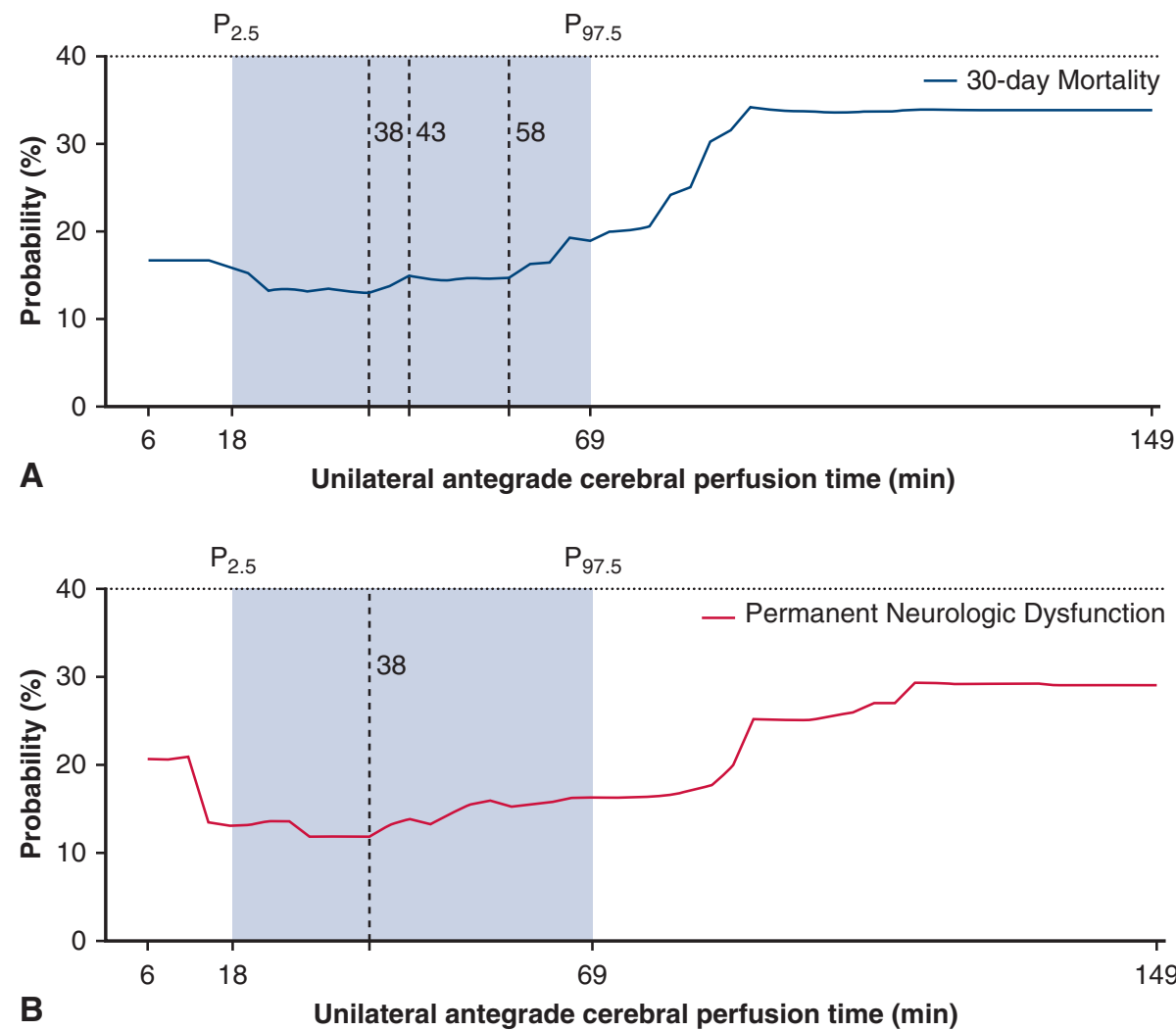

FIGURE 1. Probability for early outcomes with prolonged unilateral antegrade cerebral perfusion times. A, Probability for 30-day mortality with prolonged unilateral antegrade cerebral perfusion times. An upward trend was steep from 38 to 43 minutes and stayed at this level until 58 minutes. Then this trend became sharp again. B, Probability for permanent neurologic dysfunction with prolonged unilateral antegrade cerebral perfusion times. An upward trend was observed when unilateral antegrade cerebral perfusion time was more than 38 minutes. The blue areas are from $2.5 \%$ to $97.5 \%$, representing $95 \%$ of the patients. $P 2.5,2.5$ th percentile; $P 97.5,97.5$ th percentile.

(Figure 2,C). No obvious trends for 30-day mortality and CRRT were observed from 12 minutes (the 2.5 th percentile) to 54 minutes (the 97.5th percentile) (Figure 2, $A$ and $B$ ).

\section{Nasopharyngeal Temperature}

In the RFR, a slow downward trend was observed in 30day mortality when nasopharyngeal temperature was lower than $24.3^{\circ} \mathrm{C}$. However, when the temperature was higher than $25.0^{\circ} \mathrm{C}$, a sharp upward trend began to appear (Figure 3, A). Likewise, a slow downward trend for permanent neurologic dysfunction was observed before $24.0^{\circ} \mathrm{C}$ and then a sharp upward trend began to appear (Figure 3, B). A slow downward trend for CRRT was observed before $24.3^{\circ} \mathrm{C}$, and then a sharp upward trend began to appear (Figure 3,C). A downward trend for paraplegia was observed before $22.3^{\circ} \mathrm{C}$, and a steep upward trend was observed after $24.0^{\circ} \mathrm{C}$, with slight fluctuations (Figure $3, D$ ).

\section{DISCUSSION}

In conducting the present retrospective study, we analyzed data from the largest reported cohort of patients who have undergone aortic arch surgery with definite hypothermic circulatory arrest and unilateral antegrade cerebral perfusion, including clinical status, demographic data, surgical procedures, circulatory management strategies, cerebral protection strategies, and clinical outcomes (30day mortality, the incidence of permanent neurologic dysfunction, and the use rate of CRRT) associated with these surgical procedures. The results showed that advanced age, DeBakey type I dissection, poor cardiac function, preoperative coma, CABG, extra-anatomic bypass, and prolonged $\mathrm{CPB}$ time were independent risk factors for 30-day mortality. Advanced age, DeBakey type I dissection, and prolonged CPB time were independent risk factors for permanent neurologic dysfunction. The day of operation, advanced age, previous chronic renal dysfunction, valve surgery, and prolonged CPB time were independent risk factors for CRRT. Additionally, the results of the RFR analyses showed that the risk for 30-day mortality and permanent neurologic dysfunction began to increase when unilateral antegrade cerebral perfusion time was longer than 38 minutes. The risk for 

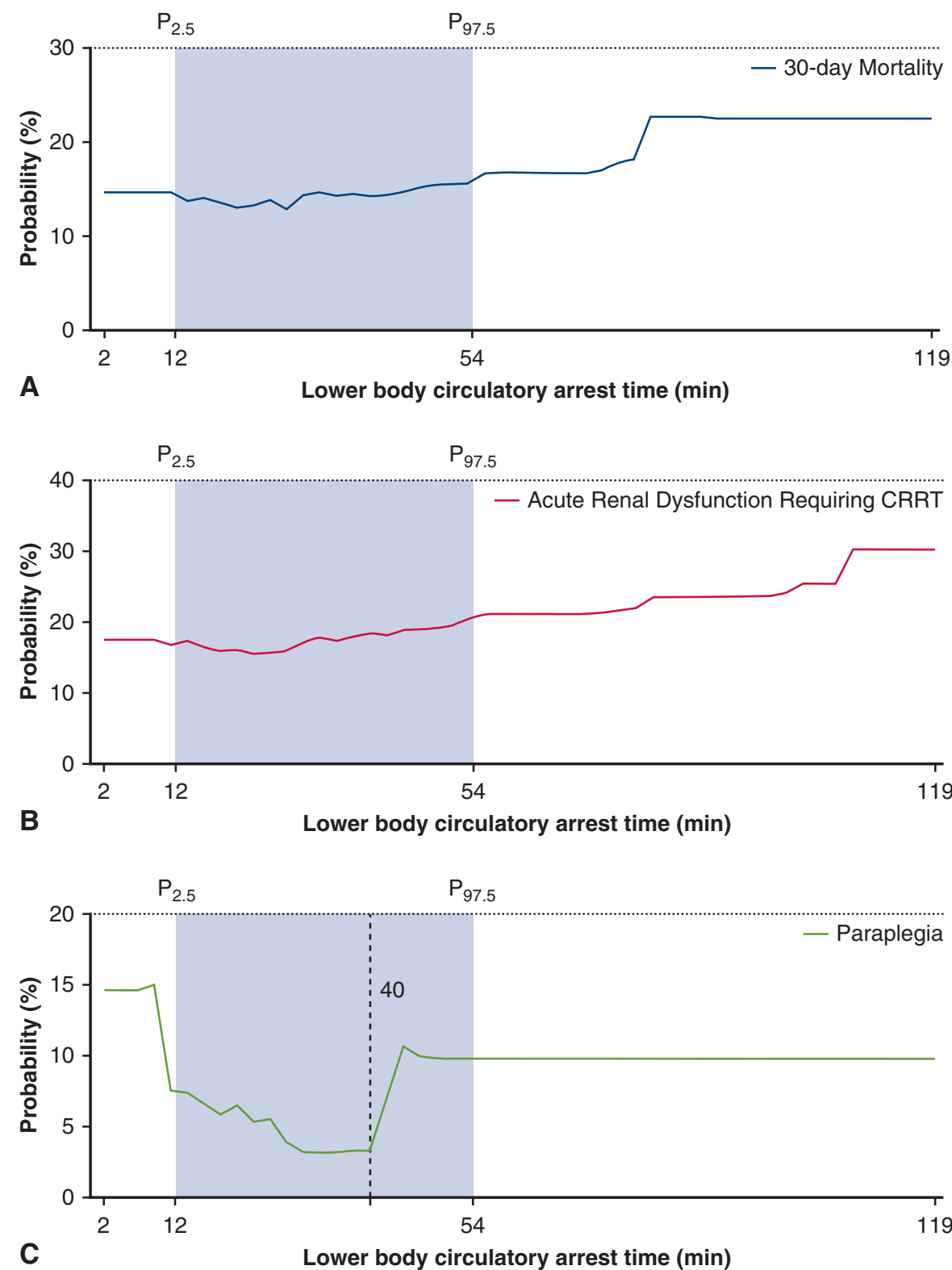

FIGURE 2. Probability for early outcomes with prolonged LBCA times. A, Probability for 30-day mortality with prolonged LBCA times. No obvious trend was observed in $95 \%$ of patients. B, Probability for acute renal dysfunction requiring CRRT with prolonged LBCA times. No obvious trend was observed in $95 \%$ of patients. C, Probability for paraplegia with prolonged LBCA times. A sharp upward trend was observed when LBCA time was longer than 40 minutes. The blue areas are from $2.5 \%$ to $97.5 \%$, representing $95 \%$ of the patients. $P 2.5,2.5$ th percentile; $P 97.5$, 97.5 th percentile; $C R R T$, continuous renal replacement therapy.

permanent neurologic dysfunction, CRRT, and paraplegia increased when the temperature was higher than approximately $24^{\circ} \mathrm{C}$.

The etiology of aortic disease and patient demographics in our study varied significantly from other studies. The mean age of all patients was lower, and the proportion of male patients was higher. ${ }^{4}$ Only $7.9 \%$ of patients experienced an aortic aneurysm, and most of them had an aortic dissection. The proportion of patients presenting with aortic aneurysm was significantly lower than the percentage in other studies. ${ }^{9}$ These results might be attributed to different racial backgrounds, high incidence of hypertension, and low rates of hypertension control ${ }^{10,11}$ in China. Although the patients in our study were younger, the proportion of patients with hypertension was similar to the percentage reported in other studies. ${ }^{12-14}$ The percentage of patients diagnosed with Marfan syndrome $(3 \%)$ was lower than reported in other studies. ${ }^{15,16}$ The incidences of coronary artery disease, diabetes, and renal failure were lower, which might be due to the lower mean age. ${ }^{17,18}$ 


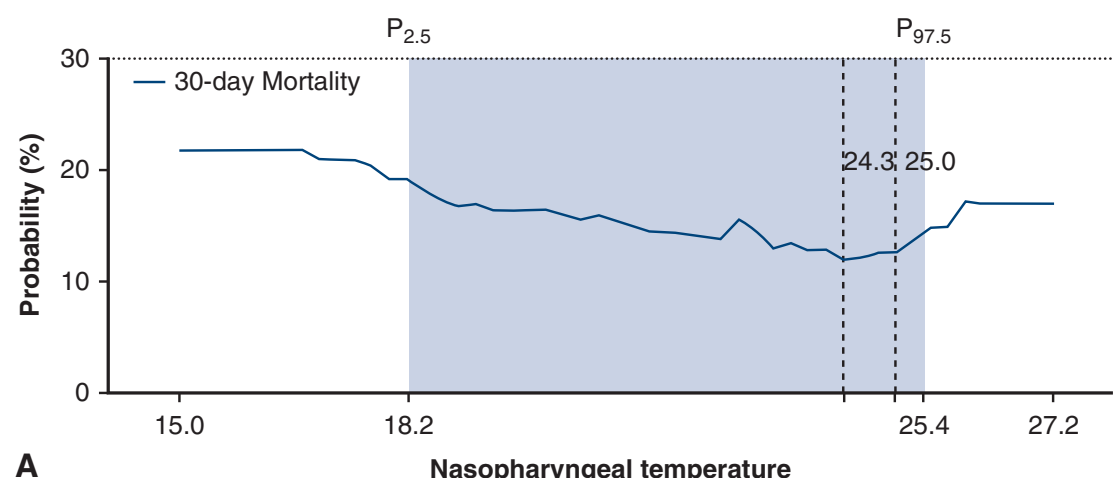

A

Nasopharyngeal temperature

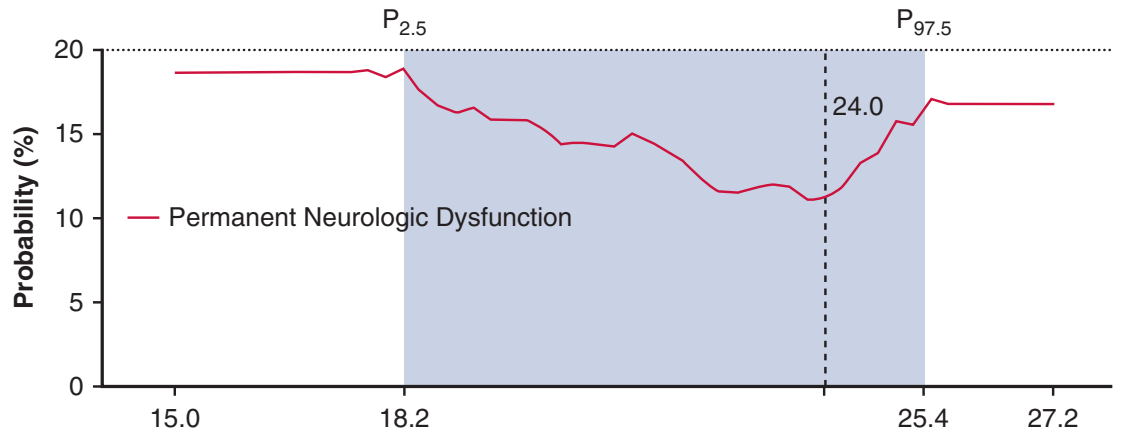

B

Nasopharyngeal temperature

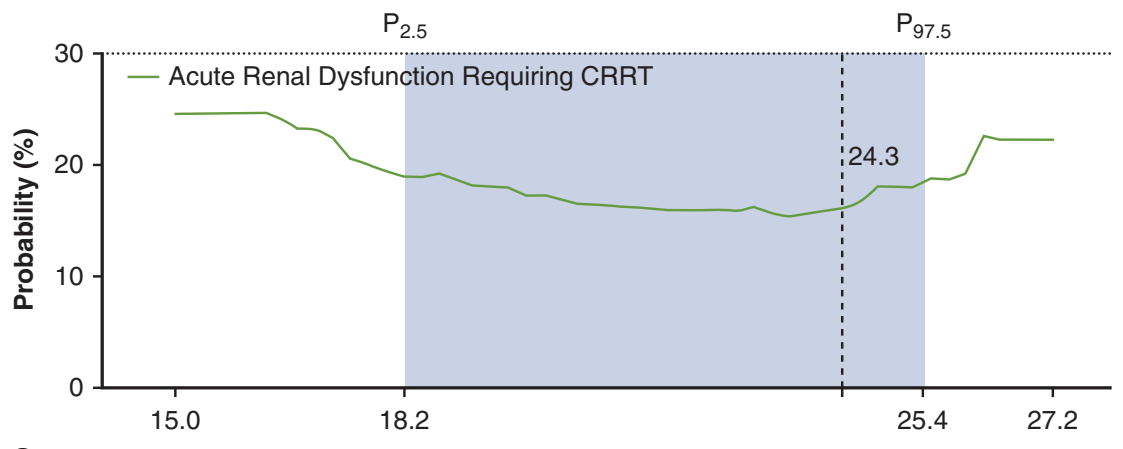

C

Nasopharyngeal temperature

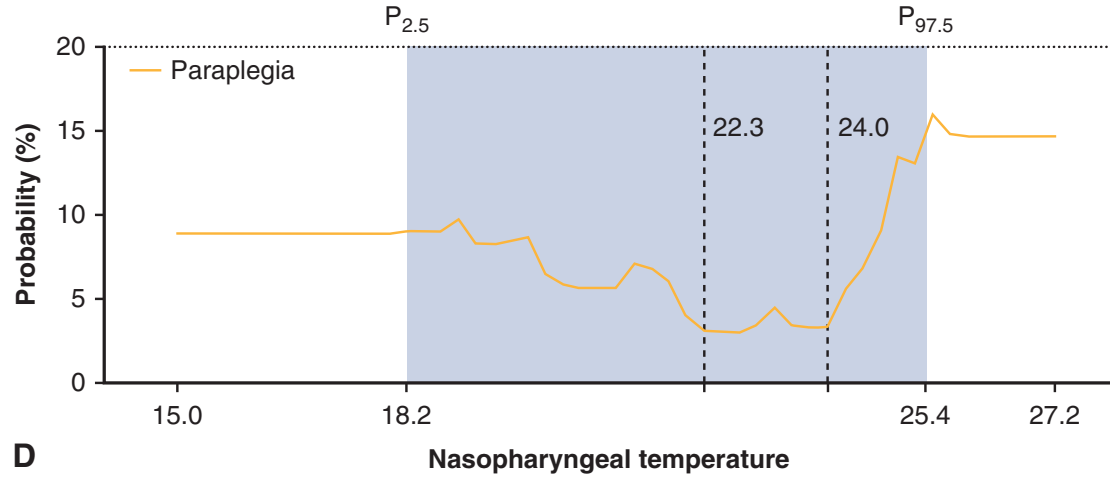

FIGURE 3. Probability for early outcomes with increased nasopharyngeal temperatures. A, Probability for 30-day mortality with increased nasopharyngeal temperatures. A slow downward trend was observed before $24.3^{\circ} \mathrm{C}$, and an upward trend was observed after $25.0^{\circ} \mathrm{C}$. B, Probability for permanent neurologic dysfunction with increased nasopharyngeal temperatures. A slow downward trend was observed before $24.0^{\circ} \mathrm{C}$, and an upward trend was observed after $24.0^{\circ} \mathrm{C}$. C, Probability for acute renal dysfunction requiring CRRT with increased nasopharyngeal temperatures. A slow downward trend was observed before $24.3^{\circ} \mathrm{C}$, and an upward trend was observed after $24.3^{\circ} \mathrm{C}$. D, Probability for paraplegia with increased nasopharyngeal temperatures. A downward trend was observed before $22.3^{\circ} \mathrm{C}$, and a steep upward trend was observed after $24.0^{\circ} \mathrm{C}$, with slight fluctuations. The blue areas are from $2.5 \%$ to $97.5 \%$, representing $95 \%$ of the patients. $P 2.5,2.5$ th percentile; $P 97.5,97.5$ th percentile; $C R R T$, continuous renal replacement therapy. 
Thirty-day mortality $(6.1 \%)$ and the rate of permanent neurologic dysfunction $(4.8 \%)$ in our study were comparable with the rates observed in other case series reported in the literature (mortality, $8 \%-15.9 \%$; rate of permanent neurologic dysfunction, $6 \%-10.5 \%)^{3,16-18}$ Similar to previous studies, in our study, the multivariable analysis identified advanced age,${ }^{19,20}$ poor cardiac function, ${ }^{21}$ preoperative coma, ${ }^{22} \mathrm{CABG},{ }^{23}$ and prolonged CPB time ${ }^{21,24}$ as independent risk factors for mortality. Advanced age $^{20,25}$ and prolonged CPB time ${ }^{21,24}$ were also independent risk factors for permanent neurologic dysfunction. Additionally, advanced age, ${ }^{19,23,24}$ preoperative chronic renal dysfunction, ${ }^{26}$ and prolonged CPB time ${ }^{22}$ were independent risk factors for CRRT.

The reported incidence of paraplegia in patients who underwent frozen elephant trunk appeared to be significantly higher than in those who were treated with the conventional elephant trunk procedure $(7.5 \%-21.7 \%$ vs $1.8 \%-5.6 \%){ }^{27-30}$ In the present retrospective study, most of the patients underwent frozen elephant trunk and total arch replacement, but the incidence of paraplegia $(2.0 \%)$ was lower than in other case series focusing on frozen elephant trunk reported in the literature. We did not find that frozen elephant trunk and total arch replacement increased the risk for paraplegia in traditional logistic regression and RFR analyses, unlike the study by Leontyev and colleagues. ${ }^{29}$ One factor that differed from other studies discussing frozen elephant trunk procedures was the LBCA time, which was significantly shorter in the present retrospective study. Roselli and colleagues $^{31}$ reported no cases of paraplegia in their series of 17 patients who underwent frozen elephant trunk procedures, with an average LBCA time comparable to the time reported in the present retrospective study. Some studies have raised concerns about the safety of the spinal cord in relation to LBCA. This study also found that the risk for paraplegia increased when LBCA time was longer than 40 minutes. Thus, the lower incidence of paraplegia could be explained by the shorter LBCA time in our center, a consequence of the unique surgical procedure we have implemented.

A large number of existing studies ${ }^{3,17,19,23}$ suggested that LBCA time had a significant effect on short-term mortality or permanent neurologic dysfunction. However, we did not observe a similar effect in traditional logistic regression and RFR analyses. Several factors could explain the difference in findings. Previous studies ${ }^{3}$ had more confounding factors because they were multicenter studies or involved multiple surgical teams. Our analysis consists of a single-center study, involving surgical procedures performed by the same surgical team. In addition, the LBCA time was significantly shorter and had smaller individual differences across the cohort in this study than in previous studies ${ }^{18,19}$ because of the surgical protocol of our center. Most of the LBCA times were within the safe time limit reported previously $y^{3,32}$ and did not have an impact on prognosis. Most patients who underwent prolonged LBCA were in low numbers and scattered throughout the cohort.

In addition to the effects of LBCA time, previous studies $^{3,24,25}$ have reported that prognosis was significantly influenced by the unilateral antegrade cerebral perfusion time. In our study, the RFR analysis showed there is a safety threshold (38 minutes) of unilateral antegrade cerebral perfusion time in relation to 30-day mortality and permanent neurologic dysfunction. Thus, we consider unilateral antegrade cerebral perfusion time an important predictor for short-term adverse outcomes during aortic arch surgery, especially when longer than 38 minutes. However, the causes of this result remain unclear. In-depth studies should further explore the mechanisms underlying the relationship between unilateral antegrade cerebral perfusion duration and adverse events.

In recent years, research has focused on temperature management strategies during LBCA. Like previous studies $^{24,33}$ that found benefits to higher temperature management strategies, our study confirmed that an increase in temperature during LBCA was related to a decrease in 30-day mortality or in incidence of permanent neurologic dysfunction in the RFR analysis. This may be related to a reduction in CPB times, postoperative bleeding, endothelial dysfunction, neuronal apoptosis, and postoperative pulmonary complications, which are typically associated with deep hypothermia. ${ }^{9,17,18,34}$ However, the benefit was demonstrated only below the safety threshold for temperature $\left(\sim 24^{\circ} \mathrm{C}\right)$, because the risks for permanent neurologic dysfunction, paraplegia, and acute renal dysfunction requiring CRRT increased with temperatures above the safety threshold. Other studies ${ }^{14,24,32}$ have reported that higher temperature circulatory management may carry a risk of spinal cord and visceral ischemia in patients undergoing prolonged LBCA. Our study confirmed that higher nasopharyngeal temperature $\left(>24^{\circ} \mathrm{C}\right)$ correlates with a higher incidence of postoperative paraplegia and acute renal dysfunction requiring CRRT in the RFR analyses. However, largerscale, multicenter studies are necessary to confirm these results and to clarify whether the increased risk associated with higher nasopharyngeal temperature occurs only in patients who undergo prolonged LBCA.

\section{Study Limitations}

Our study had several limitations. First, this study was subject to the limitations inherent to any retrospective, observational study from a single center, such as missing data, bias due to memory errors, and lack of representativeness. Some of the necessary variables were not found in the retrospective database. Second, a previous study has pointed out that neurologic evaluation is inadequate in retrospective studies. ${ }^{35}$ In this study, permanent neurologic 
dysfunction might be underestimated because neurologic evaluations and neuroimaging were not performed in patients in whom the neurologist did not observe symptoms suggestive of postoperative stroke or paraplegia. Third, in our center, LBCA time was shorter and individual differences in LBCA time were smaller compared with other studies. Moreover, most of the LBCA times were within the safe time limit reported previously., ${ }^{3,32}$ Shorter LBCA duration might explain the lack of correlation between LBCA time and 30-day mortality or CRRT. Fourth, although this study included a large sample, some critical cases might have been excluded. In cases of acute type A aortic dissection, the median time from onset to diagnosis was 2 (1-4) days. The rate of surgical intervention with 24 hours was also lower than the rate reported in other studies. ${ }^{3}$ Our center is the largest referral center for aortic disease in China. In some cases, timely treatment was impossible because of delayed transportation to our hospital. Finally, although acute, subacute, and chronic aortic dissection accounted for most cases, the causes of aortic arch surgery were heterogeneous throughout the cohort. However, we performed sensitivity analyses to explore the effects of different pathological types on outcomes.

\section{CONCLUSIONS}

In this single-center study, we reported the clinical experience associated with aortic arch surgery, including patient demographics, surgical procedures, circulatory management strategies, cerebral protection strategies, and clinical outcomes over the span of 7 years. When used as a cerebral protection strategy, unilateral antegrade cerebral perfusion may have a safety threshold of 38 minutes in relation to 30-day mortality and permanent neurologic dysfunction. Moderate hypothermia proved to be a safe and effective circulatory management strategy. However, temperatures greater than approximately $24^{\circ} \mathrm{C}$ should be used cautiously to minimize the risk for postoperative permanent neurologic dysfunction, paraplegia, and acute renal dysfunction requiring CRRT.

\section{Conflict of Interest Statement}

Authors have nothing to disclose with regard to commercial support.

The authors thank Wei Pan, who provided advice on the structure of the manuscript.

\section{References}

1. Erbel R, Aboyans V, Boileau C, Bossone E, Bartolomeo RD, Eggebrecht H, et al. 2014 ESC guidelines on the diagnosis and treatment of aortic diseases: document covering acute and chronic aortic diseases of the thoracic and abdominal aorta of the adult. The task force for the diagnosis and treatment of aortic diseases of the European Society of Cardiology (ESC). Eur Heart J. 2014;35:2873-926.

2. Hagan PG, Nienaber CA, Isselbacher EM, Bruckman D, Karavite DJ, Russman PL, et al. The International Registry of Acute Aortic Dissection (IRAD): new insights into an old disease. JAMA. 2000;283:897-903.
3. Kruger T, Weigang E, Hoffmann I, Blettner M, Aebert H. Cerebral protection during surgery for acute aortic dissection type A: results of the German Registry for Acute Aortic Dissection Type A (GERAADA). Circulation. 2011;124: 434-43.

4. Pape LA, Awais M, Woznicki EM, Suzuki T, Trimarchi S, Evangelista A, et al. Presentation, diagnosis, and outcomes of acute aortic dissection: 17-year trends from the International Registry of Acute Aortic Dissection. J Am Coll Cardiol. 2015;66:350-8.

5. Sun L, Qi R, Zhu J, Liu Y, Zheng J. Total arch replacement combined with stented elephant trunk implantation: a new "standard" therapy for type a dissection involving repair of the aortic arch? Circulation. 2011;123:971-8.

6. Ma WG, Zhu JM, Zheng J, Liu YM, Ziganshin BA, Elefteriades JA, et al. Sun's procedure for complex aortic arch repair: total arch replacement using a tetrafurcate graft with stented elephant trunk implantation. Ann Cardiothorac Surg. 2013;2:642-8.

7. Bossone E, Corteville DC, Harris KM, Suzuki T, Fattori R, Hutchison S, et al. Stroke and outcomes in patients with acute type A aortic dissection. Circulation. 2013;128(11 Suppl 1):S175-9.

8. Raja S, Rice TW, Ehrlinger J, Goldblum JR, Rybicki LA, Murthy SC, et al. Importance of residual primary cancer after induction therapy for esophageal adenocarcinoma. J Thorac Cardiovasc Surg. 2016;152:756-61.e5.

9. Leshnower BG, Myung RJ, Kilgo PD, Vassiliades TA, Vega JD, Thourani VH, et al. Moderate hypothermia and unilateral selective antegrade cerebral perfusion: a contemporary cerebral protection strategy for aortic arch surgery. Ann Thorac Surg. 2010;90:547-54.

10. Czerny M, Krahenbuhl E, Reineke D, Sodeck G, Englberger L, Weber A, et al. Mortality and neurologic injury after surgical repair with hypothermic circulatory arrest in acute and chronic proximal thoracic aortic pathology: effect of age on outcome. Circulation. 2011;124:1407-13.

11. Peacock E, Krousel-Wood M. Adherence to antihypertensive therapy. Med Clin North Am. 2017;101:229-45.

12. Ergin MA, Galla JD, Lansman SL, Quintana C, Bodian C, Griepp RB. Hypothermic circulatory arrest in operations on the thoracic aorta. Determinants of operative mortality and neurologic outcome. J Thorac Cardiovasc Surg. 1994;107:788-99.

13. Liu H, Chang Q, Zhang H, Yu C. Predictors of adverse outcome and transient neurological dysfunction following aortic arch replacement in 626 consecutive patients in China. Heart Lung Circ. 2017;26:172-8.

14. Pacini D, Pantaleo A, Di Marco L, Leone A, Barberio G, Murana G, et al. Visceral organ protection in aortic arch surgery: safety of moderate hypothermia. Eur J Cardiothorac Surg. 2014;46:438-43.

15. Berretta P, Patel HJ, Gleason TG, Sundt TM, Myrmel T, Desai N, et al. IRAD experience on surgical type A acute dissection patients: results and predictors of mortality. Ann Cardiothorac Surg. 2016;5:346-51.

16. Zierer A, Detho F, Dzemali O, Aybek T, Moritz A, Bakhtiary F. Antegrade cerebral perfusion with mild hypothermia for aortic arch replacement: single-center experience in 245 consecutive patients. Ann Thorac Surg. 2011;91:1868-73.

17. Comas GM, Leshnower BG, Halkos ME, Thourani VH, Puskas JD, Guyton RA, et al. Acute type a dissection: impact of antegrade cerebral perfusion under moderate hypothermia. Ann Thorac Surg. 2013;96:2135-41.

18. Leshnower BG, Thourani VH, Halkos ME, Sarin EL, Keeling WB, Lamias MJ, et al. Moderate versus deep hypothermia with unilateral selective antegrade cerebral perfusion for acute type A dissection. Ann Thorac Surg. 2015;100:1563-9.

19. Preventza O, Simpson KH, Cooley DA, Cornwell L, Bakaeen FG, Omer S, et al. Unilateral versus bilateral cerebral perfusion for acute type A aortic dissection. Ann Thorac Surg. 2015;99:80-7.

20. Safi HJ, Miller CC III, Lee TY, Estrera AL. Repair of ascending and transverse aortic arch. J Thorac Cardiovasc Surg. 2011;142:630-3.

21. Okada K, Omura A, Kano H, Sakamoto T, Tanaka A, Inoue T, et al. Recent advancements of total aortic arch replacement. J Thorac Cardiovasc Surg. 2012; 144:139-45.

22. Numata S, Tsutsumi Y, Monta O, Yamazaki S, Seo H, Sugita R, et al. Aortic arch repair with antegrade selective cerebral perfusion using mild to moderate hypothermia of more than 28 degrees C. Ann Thorac Surg. 2012;94:90-6.

23. Zierer A, El-Sayed Ahmad A, Papadopoulos N, Moritz A, Diegeler A, Urbanski PP. Selective antegrade cerebral perfusion and mild (28 degrees C-30 degrees C) systemic hypothermic circulatory arrest for aortic arch replacement: results from 1002 patients. J Thorac Cardiovasc Surg. 2012;144:1042-9.

24. Kamiya H, Hagl C, Kropivnitskaya I, Bothig D, Kallenbach K, Khaladj N, et al. The safety of moderate hypothermic lower body circulatory arrest with selective cerebral perfusion: a propensity score analysis. J Thorac Cardiovasc Surg. 2007; 133:501-9. 
25. Preventza O, Garcia A, Tuluca A, Henry M, Cooley DA, Simpson K, et al. Innominate artery cannulation for proximal aortic surgery: outcomes and neurological events in 263 patients. Eur J Cardiothorac Surg. 2015;48:937-42.

26. Nota H, Asai T, Suzuki T, Kinoshita T, Ikegami H, Takashima N. Risk factors for acute kidney injury in aortic arch surgery with selective cerebral perfusion and mild hypothermic lower body circulatory arrest. Interact Cardiovasc Thorac Surg. 2014;19:955-61.

27. Di Eusanio M, Borger M, Petridis FD, Leontyev S, Pantaleo A, Moz M, et al, Conventional versus frozen elephant trunk surgery for extensive disease of the thoracic aorta. J Cardiovasc Med (Hagerstown). 2014;15:803-9.

28. Etz CD, Plestis KA, Kari FA, Luehr M, Bodian CA, Spielvogel D, et al. Staged repair of thoracic and thoracoabdominal aortic aneurysms using the elephant trunk technique: a consecutive series of 215 first stage and 120 complete repairs. Eur J Cardiothorac Surg. 2008;34:605-15.

29. Leontyev S, Borger MA, Etz CD, Moz M, Seeburger J, Bakhtiary F, et al. Experience with the conventional and frozen elephant trunk techniques: a singlecentre study. Eur J Cardiothorac Surg. 2013;44:1076-83.

30. Shrestha M, Martens A, Kruger H, Maeding I, Ius F, Fleissner F, et al. Total aortic arch replacement with the elephant trunk technique: single-centre 30-year results. Eur J Cardiothorac Surg. 2014;45:289-96.
31. Roselli EE, Rafael A, Soltesz EG, Canale L, Lytle BW. Simplified frozen elephant trunk repair for acute DeBakey type I dissection. J Thorac Cardiovasc Surg. 2013;145(3 Suppl):S197-201.

32. Luehr M, Bachet J, Mohr FW, Etz CD. Modern temperature management in aortic arch surgery: the dilemma of moderate hypothermia. Eur J Cardiothorac Surg. 2014;45:27-39.

33. Preventza O, Coselli JS, Garcia A, Kashyap S, Akvan S, Simpson KH, et al. Moderate hypothermia at warmer temperatures is safe in elective proximal and tota arch surgery: results in 665 patients. J Thorac Cardiovasc Surg. 2017;153: 1011-8.

34. Algarni KD, Yanagawa B, Rao V, Yau TM. Profound hypothermia compared with moderate hypothermia in repair of acute type A aortic dissection. J Thorac Cardiovasc Surg. 2014;148:2888-94.

35. Leon MB, Smith CR, Mack MJ, Makkar RR, Svensson LG, Kodali SK, et al Transcatheter or surgical aortic-valve replacement in intermediate-risk patients. N Engl J Med. 2016;374:1609-20.

Key Words: aortic arch surgery, cerebral protection strategy, unilateral antegrade cerebral perfusion 


\section{APPENDIX E1. PRINCIPLES AND DETAILS OF STATISTIC METHOD \\ Details of Missing Data Imputation}

Missing data are shown in Table 1. Random Forest imputation was used to maximize use of available data. We imputed values in predictor data using proximity from the "randomForest" package. The algorithm starts by imputing missing values using the median. The randomForest is called with completed data. The proximity matrix from the randomForest is used to update the imputation of the missing values. For continuous predictors, the imputed value is the weights average of the nonmissing observations, where the weights are the proximities. For categoric predictors, the imputed value is the category with the largest average proximity. This process is iterated times. ${ }^{\mathrm{E} 1}$

\section{Principles and Details of Random Forest}

We used RFR with R software as the analytic strategy in part to avoid restrictive parametric modeling assumptions, given no prior knowledge of what relationships might exist, and in part because some studies previously demonstrated with this method that there is a complex interplay between characteristics of diseases and outcomes. A random Forest is a collection of decision-tree analyses, wherein a variable is chosen to optimally split the population to improve prediction. This process is applied recursively to create a tree (recursive partitioning, classification, and regression trees). Individual trees "grown" by this method are inherently unstable, which can be mitigated by creating a collection of trees from bootstrap samples of the original data set (the bootstrap data set is formed by random sampling of patients with replacement until a data set of equal size is generated; some patients will be duplicated, and an average of $37 \%$ will not be sampled). Subsequently, an ensemble average can be formulated across this Forest of individual trees. Validity of the Forest is evaluated by assessing outcomes of patients who were not selected in the bootstrap process, resulting in internal multifold cross validation. This transforms variables associated with an outcome of interest into predictors of that outcome.

We have used "randomForest" package to perform random Forest. A random Forest was constructed with 1500 trees. At each node, all variables (the day of operation, sex, age, body mass index, pathological, DeBakey type,
NYHA score, left ventricular ejection fraction, coronary artery disease, hypertension, diabetes mellitus, chronic kidney disease, Marfan syndrome, pericardial tamponade, acute heart failure, coma, lower-limb malperfusion, tracheotomy, previous CRRT, previous stroke, redo sternotomy, cannulation site of unilateral antegrade cerebral perfusion, main surgery procedure, ascending aorta replacement, Bentall, CABG, valve surgery, extra-anatomic bypass, nasopharyngeal temperature, rectal temperature, cerebral perfusion flow, unilateral antegrade cerebral perfusion time, crossclamp time, CPB, LBCA time, rewarming time) were used, but 6 candidate variables were randomly selected from among these for splitting each node (which avoids the statistical problem related to the order in which variables are incorporated into models). The reasons why we chose 1500 trees and 6 candidate variables were that the model maintained stable and the error was minimal at that moment, respectively.

\section{The Variable Importance}

We have used the "randomForest" package to measure the variable importance. The variable importance measure was the total decrease in node impurities from splitting on the variable, averaged over all trees. For classification, the node impurity is measured by the Gini index. For regression, it is measured by residual sum of squares.

\section{Partial Dependence Plot}

We used partial dependence to determine how variables were related to outcomes. Partial dependency adjusted for all variables in the model. Conditional partial dependence plots were used to detect interactions between variables (LBCA time, unilateral antegrade cerebral perfusion time, and nasopharyngeal temperature) and the outcomes (30-day mortality, permanent neurologic dysfunction, paraplegia, and acute renal dysfunction requiring CRRT). Partial dependence (logits/log odds) was calculated by randomForest package. Then we converted logit units to probability using the formula $\mathrm{P}=1 / 1[\exp (-\mathrm{z})]$. " $\mathrm{z}$ " is the logit units that we have plotted.

\section{E-Reference}

E1. Breiman L. Manual for setting up, using, and understanding Random Forest V4.0. Available at: https://www.stat.berkeley.edu/ breiman/Using_random_ forests_v4.0.pdf. Accessed May 20, 2018. 
30-day mortality

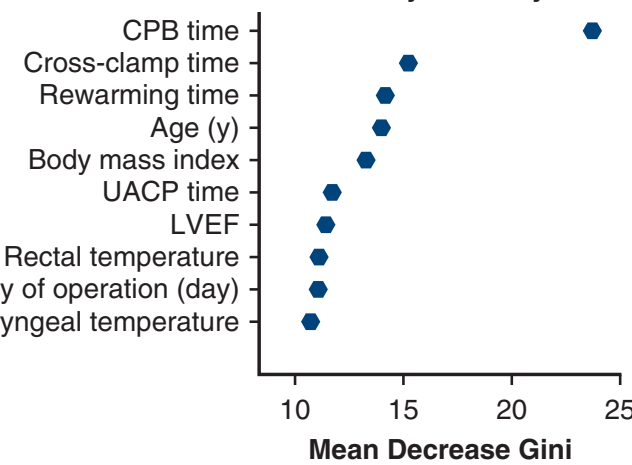

A

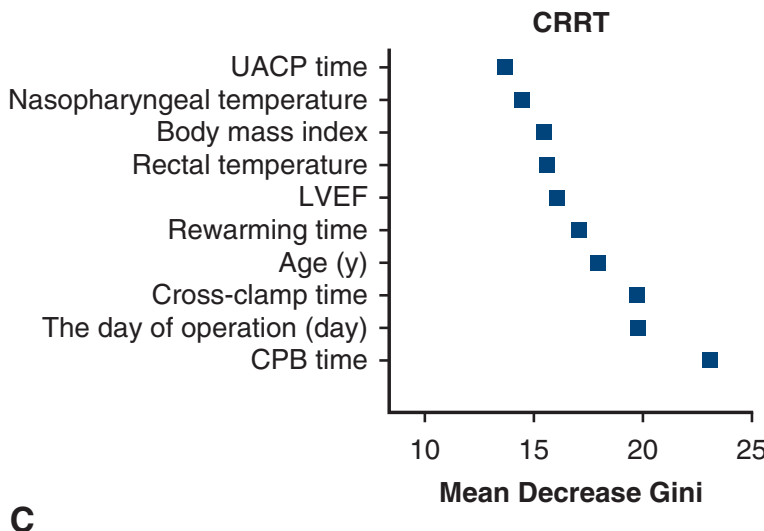

PND

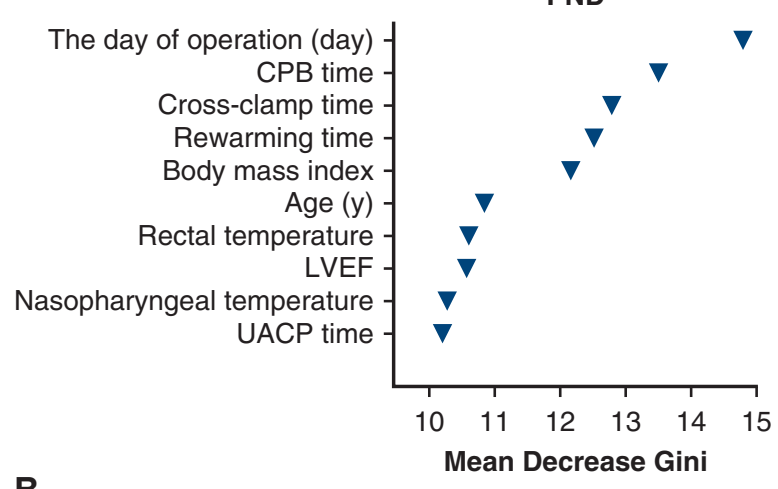

B

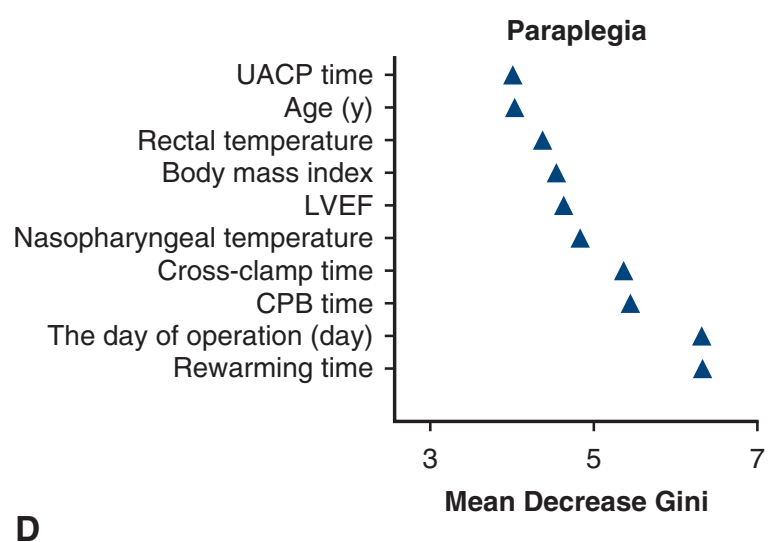

FIGURE E1. The variable importance for outcomes. A, The variable importance for 30-day mortality. B, The variable importance for permanent neurologic dysfunction. C, The variable importance for acute renal dysfunction requiring CRRT. D, The variable importance for paraplegia. $C P B$, Cardiopulmonary bypass; $U A C P$, unilateral antegrade cerebral perfusion; $L V E F$, left ventricular ejection fraction; $P N D$, permanent neurologic dysfunction; $C R R T$, continuous renal replacement therapy. 
TABLE E1. Test for collinearity of variables planned to be included in the multivariable model for 30-day mortality

\begin{tabular}{lcr}
\hline \multicolumn{1}{c}{ Variables } & Tolerance & VIF \\
\hline Age (y) & 0.94 & 1.07 \\
Acute aortic dissection & 0.82 & 1.21 \\
\hline DeBakey type & 0.03 & 32.41 \\
NYHA & 0.97 & 1.03 \\
\hline Coronary artery disease & 0.84 & 1.19 \\
Chronic kidney disease & 0.91 & 1.10 \\
\hline Pericardial tamponade & 0.95 & 1.05 \\
Coma & 0.99 & 1.01 \\
\hline Previous CRRT & 0.93 & 1.08 \\
Main surgical procedure & 0.03 & 32.89 \\
\hline CABG & 0.80 & 1.25 \\
Extra-anatomic bypass & 0.84 & 1.19 \\
\hline Nasopharyngeal temperature & 0.61 & 1.65 \\
Rectal temperature & 0.58 & 1.72 \\
\hline UACP time & 0.64 & 1.56 \\
Crossclamp time & 0.31 & 3.18 \\
\hline CPB time & 0.25 & 3.95 \\
\hline Rewarming time & 0.48 & 2.09 \\
\hline VIF, Vain
\end{tabular}

VIF, Variance Inflation Factor; NYHA, New York Heart Association; CRRT, continuous renal replacement therapy; $C A B G$, coronary artery bypass grafting; $U A C P$, unilateral antegrade cerebral perfusion; $C P B$, cardiopulmonary bypass.

TABLE E2. Test for collinearity of variables planned to be included in the multivariable model for postoperative permanent neurologic dysfunction

\begin{tabular}{lcr}
\hline \multicolumn{1}{c}{ Variables } & Tolerance & VIF \\
\hline Time $(\mathrm{d})$ & 0.71 & 1.40 \\
Age $(\mathrm{y})$ & 0.91 & 1.09 \\
\hline Acute aortic dissection & 0.82 & 1.21 \\
\hline DeBakey type & 0.03 & 32.15 \\
\hline NYHA & 0.94 & 1.06 \\
Hypertension & 0.90 & 1.11 \\
\hline Previous stroke & 0.98 & 1.02 \\
Cannulation site & 0.96 & 1.04 \\
\hline Main surgical procedure & 0.03 & 32.16 \\
CABG & 0.89 & 1.13 \\
\hline Nasopharyngeal temperature & 0.53 & 1.90 \\
Rectal temperature & 0.58 & 1.73 \\
\hline UACP time & 0.65 & 1.55 \\
Crossclamp time & 0.32 & 3.15 \\
\hline CPB time & 0.26 & 3.93 \\
Rewarming time & 0.48 & 2.09 \\
\hline VIF, Vairce &
\end{tabular}

VIF, Variance Inflation Factor; $N Y H A$, New York Heart Association; $C A B G$, Coronary artery bypass grafting; $U A C P$, unilateral antegrade cerebral perfusion; $C P B$, cardiopulmonary bypass.
TABLE E3. Test for collinearity of variables planned to be included in the multivariable model for postoperative paraplegia

\begin{tabular}{lcc}
\hline & Tolerance & VIF \\
\hline Age $(\mathrm{y})$ & 0.97 & 1.03 \\
Coronary artery disease & 0.84 & 1.20 \\
CABG & 0.81 & 1.23 \\
Crossclamp time & 0.39 & 2.59 \\
CPB time & 0.25 & 3.96 \\
Rewarming time & 0.50 & 2.02 \\
\hline
\end{tabular}

$V I F$, Variance Inflation Factor; $C A B G$, coronary artery bypass grafting; $C P B$, cardiopulmonary bypass.

TABLE E4. Test for collinearity of variables planned to be included in the multivariable model for postoperative continuous renal replacement therapy

\begin{tabular}{lcr}
\hline & Tolerance & VIF \\
\hline Time (d) & 0.82 & 1.22 \\
\hline Age $(y)$ & 0.89 & 1.12 \\
\hline Acute aortic dissection & 0.80 & 1.25 \\
\hline DeBakey type & 0.03 & 32.25 \\
\hline NYHA & 0.94 & 1.07 \\
\hline Hypertension & 0.88 & 1.14 \\
\hline Chronic kidney disease & 0.97 & 1.03 \\
\hline Pericardial tamponade & 0.97 & 1.03 \\
\hline Lower-limb malperfusion & 0.98 & 1.02 \\
\hline Main surgical procedure & 0.03 & 32.42 \\
\hline Ascending aorta replacement & 0.86 & 1.16 \\
CABG & 0.89 & 1.13 \\
\hline Valve surgery & 0.93 & 1.07 \\
Rectal temperature & 0.81 & 1.24 \\
\hline UACP time & 0.32 & 3.15 \\
\hline Crossclamp time & 0.32 & 3.13 \\
\hline CPB time & 0.27 & 3.76 \\
\hline LBCA time & 0.37 & 2.68 \\
\hline Rewarming time & 0.49 & 2.02 \\
\hline$V I F$, Variance Inflation Factor; $N Y H A$, New York Heart Association; $C A B G$, coronary \\
artery bypass grafting; $U A C P$, unilateral antegrade & cerebral perfusion; $C P B$, cardio- \\
pulmonary bypass; $L B C A$, lower-body circulatory arrest. & \\
\hline
\end{tabular}


TABLE E5. Accuracy of multivariable logistic regression models

\begin{tabular}{lccc}
\hline & & \multicolumn{2}{c}{ Hosmer-Lemeshow test } \\
\cline { 3 - 4 } \multicolumn{1}{c}{ Name } & Accuracy (\%) & Chi-square & $\boldsymbol{P}$ \\
\hline 30-d mortality (\%) & 93.85 & 3.252 & .918 \\
$\begin{array}{l}\text { Permanent neurologic } \\
\quad \text { dysfunction }\end{array}$ & 95.14 & 6.436 & .598 \\
CRRT & & & \\
Paraplegia & 92.18 & 8.802 & .359 \\
\hline
\end{tabular}

CRRT, Continuous renal replacement therapy.

TABLE E6. Accuracy of random Forest regression models

\begin{tabular}{lc}
\hline \multicolumn{1}{c}{ Name } & OBB estimate of error rate $(\%)$ \\
\hline 30-d mortality (\%) & 6.03 \\
Permanent neurologic dysfunction & 4.80 \\
CRRT & 7.82 \\
Paraplegia & 1.99 \\
\hline
\end{tabular}

$O B B$, Out-of-bag; $C R R T$, Continuous renal replacement therapy. 